\title{
Anfípodos (Crustacea: Gammaridea) del sector norte del Mar Caribe: listado faunístico, registros nuevos y distribución espacial
}

\author{
Amphipods (Crustacea: Gammaridea) in the northern sector of the Caribbean Sea: checklist, \\ new reports and spatial distribution
}

\author{
Ignacio Winfield $^{1 *}$ y Elva Escobar-Briones ${ }^{2}$ \\ ${ }^{1}$ Laboratorio de Crustáceos, FES-Iztacala-Universidad Nacional Autónoma de México, Apartado postal 314, Tlalnepantla, 54090, Estado de México, \\ México. \\ ${ }^{2}$ Unidad Académica Sistemas Oceanográficos y Costeros, ICMyL-Universidad Nacional Autónoma de México, Apartado postal 70-305 Ciudad \\ Universitaria, 04510, México D.F. \\ *Correspondencia: ignacioc@servidor.unam.mx
}

Resumen. Se estudió la composición faunística, los registros nuevos y la distribución espacial de los anfípodos bentónicos del sistema arrecifal del sector norte del Mar Caribe. Fueron identificadas 23 especies agrupadas en 9 familias y 7 superfamilias, entre los 25 y 419 m de profundidad. Las especies Ampelisca vadorum, Ampelisca verrilli y Haploops sp., constituyen registros nuevos para la región. En términos de abundancia y frecuencia las especies dominantes de la asociación fueron Leucothoe spinicarpa, Elasmopus rapax, Leucothoe sp., Grandidierella sp., Ericthonius sp. y Chevalia sp. La presencia de intersticios en los fragmentos de coral y las tormentas tropicales que depositan a profundidad dichos restos coralinos tienen influencia sobre la distribución batimétrica y geográfica de la composición, la riqueza y la abundancia de las especies de anfípodos en el área de estudio.

Palabras clave: fauna críptica, arrecife Mesoamericano, Chinchorro, Cozumel.

\begin{abstract}
The taxonomic diversity, new records, and spatial distribution of the benthic amphipods from a coral reef in the northern sector of the Caribbean Sea were analyzed. A total of 23 species, belonging to 9 families and 7 superfamilies were identified from depths ranging from 25 to $419 \mathrm{~m}$. Ampelisca vadorum, Ampelisca verrilli and Haploops sp. are new records for the area. The amphipod association was dominated by Leucothoe sp., Grandidierella sp., Ericthonius sp., Chevalia sp., Leucothoe spinicarpa, and Elasmopus rapax based on abundance and frequency. The occurrence of coral rubble deposited in deeper waters by tropical storms has an influence on the depth and geographic distribution, species richness, and abundance of the benthic amphipods.
\end{abstract}

Key words: Cryptic faunal, Mesoamerican reef, Chinchorro, Cozumel Island.

\section{Introducción}

Los anfípodos asociados a los arrecifes de coral y los fragmentos de éstos representan un grupo dominante de crustáceos en el intervalo de tallas pequeñas (de milímetros a centímetros) de este subfilo, registrándose una diversidad y abundancia elevadas por el éxito reproductivo y los patrones gregarios (Thomas, 1993b). Diversas adaptaciones morfológicas les han permitido incursionar en los espacios intersticiales, habitando en los restos de coral y en las algas. Como parte de la criptofauna coexisten en calidad de comensales de invertebrados

Recibido: 25 enero 2006; aceptado: 25 septiembre 2006 sésiles y gelatinosos, tubícolas y horadadores (Thomas, 1997; LeCroy, 2000). En el arrecife algunas especies (Hartmanodes nyei [Shoemaker, 1933], Metharpinia floridana [Shoemaker, 1933], Ampelisca schellenbergi Shoemaker, 1933) realizan migraciones en la columna de agua con fines de alimentación y reproducción (LeCroy, 2000; Cházaro-Olvera et al., 2002). El papel que desempeñan los anfípodos en los ambientes carbonatados incluye la transferencia de materia y energía (BellanSantini, 1999) promoviendo la bioturbación en contadas ocasiones en los escasos fondos blandos (Burton, 2002). En los estudios de monitoreo ambiental los anfípodos son excelentes bioindicadores en ecosistemas arrecifales (Thomas, 1993a). 
Como antecedentes de estudios en la región se pueden citar los trabajos de Shoemaker (1933, 1934, 1935, 1948) con la descripción de especies para las Antillas Mayores y los de Stephensen $(1933,1947)$ en las Antillas Menores. Diversas expediciones aportaron registros nuevos del orden Gammaridea frente a Colombia y Venezuela (Ruffo, 1950, 1954; Barnard, 1954, 1964). Los trabajos de Barnard y Thomas (1983), Galan (1984), Ortiz y Lemaitre (1994), Thomas y Ortiz (1995) y Díaz-Díaz (2001) han incrementado la información de los anfípodos bentónicos en arrecifes. Los estudios en el sector norte del Mar Caribe (NMC) se han limitado a la zona intermareal y la laguna arrecifal entre 0.3 a $15 \mathrm{~m}$ de profundidad e incluyen la costa oriental de la península de Yucatán (Markham et al., 1990; McKinney, 1977), isla Mujeres (McKinney, 1979), la Reserva de la Biosfera de Sian Ka'an (Oliva-Rivera y Jiménez-Cueto, 1992), la laguna de Yalahau (OlivaRivera y Jiménez-Cueto, 1997) y la laguna arrecifal del banco Chinchorro (Oliva-Rivera, 2003).

El presente estudio contribuye con registros nuevos de anfípodos crípticos y bentónicos del orden Gammaridea recolectados a profundidades de 25 a $409 \mathrm{~m}$ a lo largo del canal de Cozumel en el NMC. Con esta información se discute el patrón de la distribución de las especies en el ambiente sedimentario.

Área de estudio. El NMC colinda al oeste con la costa del estado de Quintana Roo, México. Los muestreos se llevaron a cabo a lo largo del canal de Cozumel en el polígono delimitado al sur por la frontera con Belice, y hasta la isla Contoy al norte, a lo largo del canal de Cozumel. Los sitios de colecta se ubicaron en el borde de los escarpes del banco Chinchorro (BC), de la isla Cozumel (IC) y del banco Arrowsmith (Fig. 1).

La expedición se llevó a cabo en las inmediaciones del arrecife Mesoamericano; el cual, en las zonas someras está formado por arrecifes de barrera y bordeante Jordán-Dahlgren, 1979, 1993; Guilcher, 1988). En aguas profundas, el Borderland del NMC representa un relieve geomorfológico complejo constituido por escarpes, trincheras, crestas montañosas y canales submarinos (Escobar-Briones, 2004).

En el Mar Caribe (MC) se han reconocido 4 masas de agua por debajo de la capa de mezcla, de las cuales el agua subsuperficial subtropical del Atlántico del norte (50 a $250 \mathrm{~m}$ de profundidad) y el agua central del Atlántico noroccidental ( 250 a 750 m de profundidad) están presentes en el área de estudio (Gallegos y Czitrom, 1997). El patrón de circulación superficial-subsuperficial en el canal de Yucatán y región afectada se describe detalladamente en Bunge et al. (2002) y está regionalmente dominado por la corriente de Yucatán (Candela et al., 2002; Escobar-

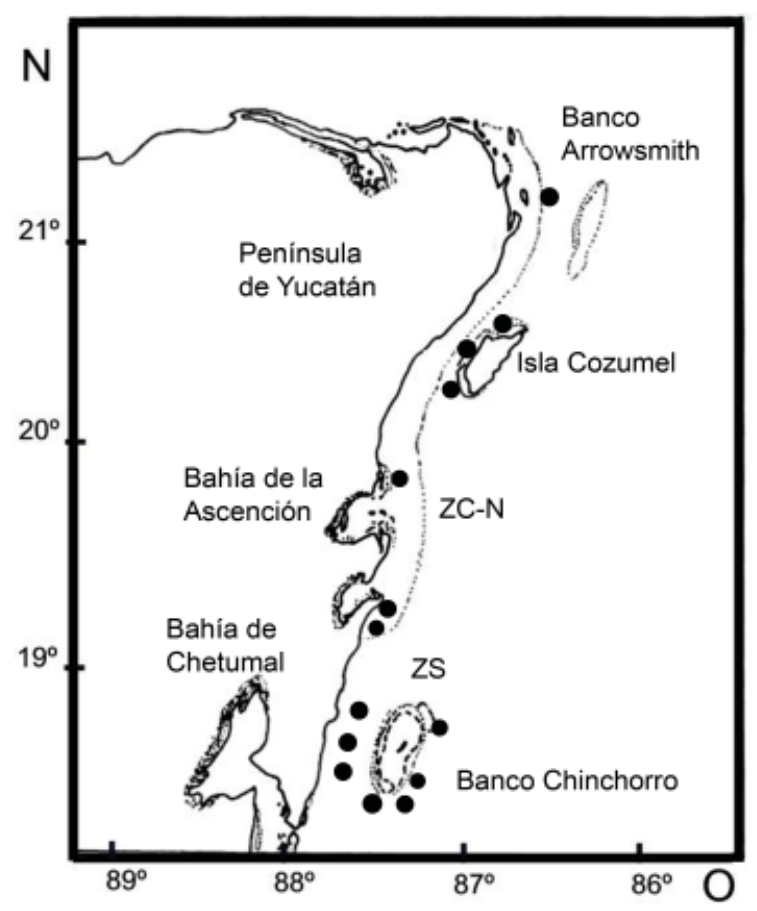

Figura 1. Toponimia del sector norte del Mar Caribe. Se muestra la ubicación de los sitios de muestreo y las zonas del sistema arrecifal: zona centro-norte (ZC-N) y zona sur (ZS). Tomado y modificado de Jordán-Dahlgren (1989a).

Briones, 2004), en su parte más somera se diferencia una trayectoria norte-noreste paralela a la costa (Merino-Ibarra, 1986).

\section{Material y métodos}

Los ejemplares se recolectaron en la campaña oceanográfica Edwin Link, realizada en agosto de 1990 a bordo del B/O Seward Johnson, como parte del proyecto de colaboración "Tropical deep reef floral and faunal studies" entre Harbor Branch Oceanographic Institution y el Instituto de Ciencias del Mar y Limnología de la Universidad Nacional Autónoma de México (EEB). El programa de muestreo incluyó 33 localidades, de las cuales 14, ubicadas entre 25 y 419 m de profundidad, presentaron anfípodos. Estas localidades se localizaron desde el BC hasta la IC (Cuadro 1). Los fragmentos y cabezos de coral de mayor tamaño se recolectaron con el brazo robótico del sumergible Johnson Sea-Link y se depositaron en una canastilla. Los sedimentos, algas y fragmentos de tamaño menor fueron recolectados por succión y depositados en los contenedores de acrílico; ambos, fragmentos grandes y pequeños, fueron transportados a la superficie al final 
Cuadro 1. Ubicación y profundidad de los sitios de muestreo con presencia de anfípodos bentónicos en el sector norte del mar Caribe (C/O Edwin Link, 1990).

\begin{tabular}{cccc}
\hline Sitio & Latitud $N$ & Longitud O & Profundidad $(\mathrm{m})$ \\
\hline 2770 & $20^{\circ} 22.31^{\prime}$ & $87^{\circ} 02.08^{\prime}$ & $25-242$ \\
2771 & $20^{\circ} 25.97^{\prime}$ & $87^{\circ} 00.97^{\prime}$ & $96-209$ \\
2772 & $20^{\circ} 39.59^{\prime}$, & $86^{\circ} 49.64^{\prime}$ & $60-216$ \\
2774 & $18^{\circ} 45.63^{\prime}$ & $87^{\circ} 15.84^{\prime}$ & $25-225$ \\
2777 & $18^{\circ} 45.58^{\prime}$ & $87^{\circ} 15.80^{\prime}$ & $32-248$ \\
2778 & $18^{\circ} 45.27^{\prime}$ & $87^{\circ} 15.87^{\prime}$ & $160-203$ \\
2780 & $18^{\circ} 30.94^{\prime}$ & $87^{\circ} 26.61^{\prime}$ & $30-262$ \\
2782 & $18^{\circ} 34.41^{\prime}$ & $87^{\circ} 26.80^{\prime}$ & $45-419$ \\
2784 & $18^{\circ} 42.06^{\prime}$ & $87^{\circ} 41.97^{\prime}$ & $25-115$ \\
2785 & $18^{\circ} 41.31^{\prime}$ & $87^{\circ} 47.01^{\prime}$ & $40-243$ \\
2786 & $18^{\circ} 20.73^{\prime}$ & $87^{\circ} 46.50^{\prime}$ & $65-300$ \\
2787 & $18^{\circ} 53.79^{\prime}$ & $87^{\circ} 36.32^{\prime}$ & $25-333$ \\
2789 & $18^{\circ} 53.95^{\prime}$ & $87^{\circ} 36.68^{\prime}$ & $37-306$ \\
2790 & $19^{\circ} 13.12^{\prime}$ & $87^{\circ} 30.31^{\prime}$ & $159-324$ \\
\hline
\end{tabular}

de la inmersión para ser procesados a bordo del buque. El material fue fraccionado en una mesa de trabajo en cubierta y tamizado con un sistema de flujo continuo. Los ejemplares de anfípodos crípticos extraídos de los cabezos de coral, de las algas y sedimento se colocaron en viales de vidrio y se preservaron con etanol al 70\%. La información ambiental y los datos sobre la inmersión fueron anotados en una bitácora para su uso posterior.

Los ejemplares fueron identificados en el laboratorio a nivel específico con base en las claves y descripciones propuestas en las publicaciones de Bousfield (1973), Barnard y Karaman (1991), Thomas y Barnard (1990), Thomas (1993b), Ortiz (1994), Lowry y Stoddart (1997), Krapp-Schickel y Jarret (2000) y LeCroy (2000, 2001). En el caso de organismos incompletos, la identificación se realizó al nivel más cercano al específico. En este estudio se adoptó la clasificación semifilética y el arreglo de las especies en superfamilias propuestos por Bousfield y Shih (1994) y Bousfield (2001). La distribución geográfica y ampliaciones del ámbito se integraron con la información de publicaciones recientes; asimismo, los registros nuevos de anfípodos bentónicos para el NMC se presentan sintética y comparativamente. El material biológico se depositó en la Colección Nacional de Crustáceos (CNCR) del Instituto de Biología, UNAM, con los códigos mencionados en la lista sistemática de los anfípodos.

En cada localidad de muestreo se cuantificó la abundancia total como el número de individuos por especie
$(N)$ y la riqueza específica como el número de especies (s). Asimismo se estimó para cada especie el Índice del Valor de Importancia (IVI) Este índice refleja el predominio de cada especie en la asociación o grupo de organismos al considerar la abundancia (N) $(100 \%)$ más la frecuencia (fi) $(100 \%)$ relativas (Brower et al., 1998). Con el porcentaje acumulado $(\leq 200 \%)$ del IVI por especie, se elaboró una curva de dominancia acumulativa para la asociación de anfípodos (Cartes et al., 2001) que permite reconocer las especies dominantes ( $\leq 100 \%$ del IVI acumulado) en la asociación de anfípodos. Para cada familia reconocida se determinó el porcentaje relativo (fi\%) a partir del porcentaje de las fi representadas por todas las especies en cada una de las familias.

La prueba $\chi^{2}(\mathrm{gl} \mathrm{n}-1, \mathrm{p}<0.05)$ fue utilizada para comprobar la continuidad o discontinuidad entre las zonas sur y centro-norte del NMC con base en la variabilidad de la composición de especies. Esta prueba no paramétrica se aplica cuando hay una distribución libre de los datos o para probar la independencia u homogeneidad entre 2 o más poblaciones de datos para una característica (Sokal y Rohlf, 1988); parámetros que se cumplen en los datos de este estudio. La matriz de datos utilizada para esta prueba incluyó las especies y los valores de abundancia total para las estaciones de las zonas sur y centro-norte del NMC.

\section{Resultados}

En este estudio se identificaron 23 especies de anfípodos bentónicos para el NMC. El arreglo taxonómico incluyó 9 familias y 7 superfamilias; 3 para el grupo Natantia y 4 para el Reptantia (sensu Bousfield, 2001), todas pertenecientes al suborden Gammaridea.

Listado sistemático de los anfípodos bentónicos:

Superorden Peracarida Calman, 1904

Orden Amphipoda Latreille, 1816

Suborden Gammaridea Latreille, 1803

Superfamilia Ampeliscoidea Bousfield, 1979

Familia Ampeliscidae Costa, 1857

1. Ampelisca sp. (CNCR10856)

2. Ampelisca bicarinata Goeke y Heard, 1983

(CNCR10748)

3. Ampelisca holmesi Pearse, 1908 (CNCR10776)

4. Ampelisca vadorum Mills, 1963 (CNCR11001)

5. Ampelisca verrilli Mills, 1967 (CNCR11072)

6. Haploops sp. (CNCR11130)

Superfamilia Corophioidea Barnard y Barnard, 1983

Familia Aoridae Stebbing, 1899

7. Bemlos sp. (CNCR10891) 
8. Grandidierella sp. (CNCR10879, 10992, 11096)

9. Rudilemboides naglei Bousfield, 1973

(CNCR11049) Familia Isaedidae Dana, 1855

10. Chevalia sp. (CNCR10853)

Familia Ischyroceridae Stebbing, 1899

11. Cerapus sp. (CNCR10807, 11196)

12. Ericthonius sp. (CNCR10853, 10879, 10996)

Superfamilia Gammaroidea Bousfield, 1977

Familia Melitidae sensu Jarret y Bousfield, 1996

13. Dulichiella appendiculata (Say, 1818)

(CNCR10877)

14. Elasmopus sp. (CNCR10996, 10753)

15. Elasmopus rapax Costa, 1853 (CNCR10945, 10891)

16. Maera cf. jerrica Krapp-Schickel y Jarret, 2000 (CNCR10877)

Superfamilia Leucothoidea Bousfield, 1979

Familia Leucothoidae Dana, 1852

17. Leucothoe sp. (CNCR10753, 11151, 10976)

18. Leucothoe spinicarpa (Abildgaard, 1789) (CNCR10779, 10771)

Superfamilia Lysianassoidea Lowry y Stoddart, 1997

Familia Lysianassidae Dana, 1849

Subfamilia Lysianassinae Dana, 1849

19. Concarnes sp. (CNCR10854)

20. Lysianopsis sp. (CNCR11064)

21. Lysianopsis alba Holmes, 1903 (CNCR11049, 10956)

Superfamilia Stenothoidea Bousfield, 2001

Familia Amphilochidae Boeck, 1871

Subfamilia Amphilochinae Barnard y Karaman, 1991

22. Gitana dominica Thomas y Barnard, 1990

(CNCR10905)

Superfamilia Synopioidea Bousfield, 1979

Familia Synopiidae Dana, 1855

23. Synopia sp. (CNCR11165)

Las superfamilias Ampeliscoidea, Corophioidea y Gammaroidea se diferenciaron por el número mayor de especies: 6 para las 2 primeras y 4 para la última. Las familias Ampeliscidae (6 spp., $N=10$, fi\% =16.7), Melitidae (4 spp., $N=7$, fi\% =16.7), Aoridae (3 spp., $N$ $=8, f i=16.7)$ y Leucothoidae ( 2 spp., $N=13$, fi $\%=16.7$ ) se reconocieron como componentes dominantes de la asociación de anfípodos con base en la riqueza específica y la abundancia total elevadas (Cuadro 2). El patrón de distribución de estas familias en el área de estudio, permitió diferenciar en la zona centro-norte $(\mathrm{ZC}-\mathrm{N})$ a la familia Ampeliscidae, particularmente en el noreste (NE) y sotavento de la IC y, para la zona sur (ZS), a Melitidae (sotavento y SO de BC), Leucothoidae (SE de BC) y Aoridae (distribución amplia) (Fig. 2).
Las 5 familias restantes, Ischyroceridae (2 spp., $N=9$ ), Lysianassidae (3 spp., $N=4$ ), Isaedidae (1 sp., $N=8$ ), Amphilochidae (1 sp., $N=1$ ) y Synopidae (1 sp., $N=1$ ), presentaron una distribución restringida al sector SO-SE de BC.

La asociación de anfípodos en el NMC estuvo dominada

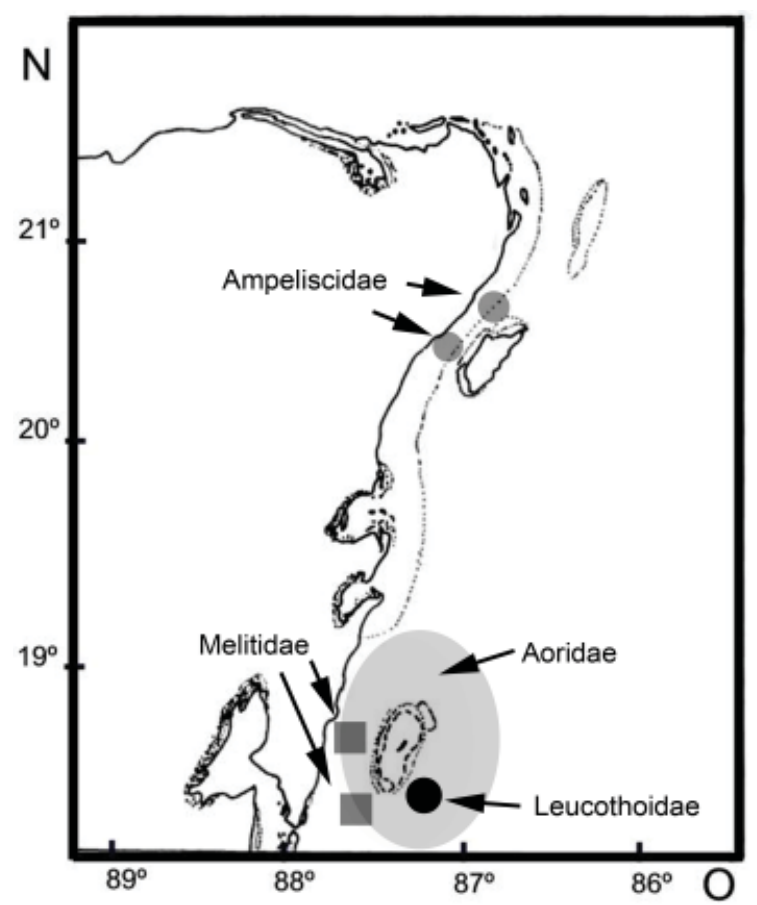

Figura 2. Distribución de las familias dominantes de los anfípodos recolectados en el sector norte del Mar Caribe. Aoridae, círculo gris claro; Leucothoidae, círculo negro; Melitidae, cuadrados; Ampeliscidae, círculos gris oscuro. Tomado y modificado de Jordán-Dahlgren (1989a).

por 6 especies. Con base en el IVI las especies: Leucothoe sp. (23.1 IVI), Grandidierella sp. (20.9 IVI), Ericthonius sp. (19.8 IVI), Chevalia sp. (15.9 IVI), Leucothoe spinicarpa (14.9 IVI) y Elasmopus rapax (10.5 IVI), constituyeron en conjunto con el $100 \%$ acumulado (Cuadro 2). Las otras 17 especies contribuyeron con el porcentaje acumulado restante (ca. 95\% del IVI) (Cuadro 2).

La ZS y ZC-N se diferenciaron con base en los valores de la riqueza de especies, y las abundancias total y relativa (Cuadro 3). La ZC-N se caracterizó por el bajo número de especies (6) y una abundancia total baja (17 ind) en la cual se reconoció la dominancia de las especies Chevalia sp. (Isaedidae) y Ericthonius sp. (Ischyroceridae) con ca. $70 \%$. La ZS presentó una riqueza de especies (19) y abundancia total (42 ind.) elevadas, con la dominancia de las especies Leucothoe sp. (Leucothoidae), Grandidierella 
Cuadro 2. Especies de anfípodos bentónicos recolectados en el sector norte del mar Caribe, incluyendo el intervalo de profundidad $(\mathrm{m})$, el porcentaje relativo de cada familia (fi\%), el número total de individuos $(N)$, la frecuencia (fi) y el índice del Valor de Importancia $(I V I)$.

\begin{tabular}{lllllll}
\hline Especies & Profundidad & fi\% & $N$ & $f i$ & IVI & \\
\hline
\end{tabular}

Suborden Gammaridea

Superfamilia Ampeliscoidea

Familia Ampeliscidae

1. Ampelisca sp.

2. Ampelisca bicarinata

3. Ampelisca holmesi

4. Ampelisca vadorum

5. Ampelisca verrilli

6. Haploops sp.

Superfamilia Corophioidea

Familia Aoridae

7. Bemlos sp.

8. Grandidierella sp.

9. Rudilemboides naglei

Familia Isaedidae

10. Chevalia sp.

Familia Ischyroceridae

11. Cerapus sp.

12. Ericthonius sp.

Superfamilia Gammaroidea

Familia Melitidae

13. Dulichiella appendiculata

14. Elasmopus sp.

15. Elasmopus rapax

16. Maera cf. jerrica

Superfamilia Leucothoidea

Familia Leucothoidae

17. Leucothoe sp.

18. Leucothoe spinicarpa

Superfamilia Lysianassoidea

Familia Lysianassidae

Subfamilia Lysianassinae

19. Concarnes sp.

20. Lysianopsis sp.

21. Lysianopsis alba

85

25

Superfamilia Stenothoidea

Familia Amphilochidae

Subfamilia Amphilochinae

22. Gitana dominica

Superfamilia Synopioidea

Familia Synopiidae

23. Synopia sp.
80

53

43

112

120

58

162

$25-45$

110

$53-60$

$32-403$

$20-185$

45

$25-162$

$89-110$

$25-162$

$53-110$

$53-80$

16.7

$\begin{array}{lll}1 & 1 & 4.4 \\ 2 & 1 & 6.1 \\ 4 & 1 & 9.3 \\ 1 & 1 & 4.4 \\ 1 & 1 & 4.4 \\ 1 & 1 & 4.4\end{array}$

16.7

$\begin{array}{llll} & 1 & 1 & 4.4 \\ & 6 & 4 & 20.9 \\ & 1 & 1 & 4.4 \\ 2.8 & & & \\ & 8 & 1 & 15.9 \\ 13.9 & & & \\ & 2 & 2 & 8.8 \\ & 7 & 3 & 19.8\end{array}$

16.7

$\begin{array}{lll}1 & 1 & 4.4 \\ 2 & 2 & 8.8 \\ 3 & 2 & 10.5 \\ 1 & 1 & 4.4\end{array}$

16.7

$\begin{array}{lll}9 & 3 & 23.1 \\ 4 & 3 & 14.9\end{array}$

11.1

$\begin{array}{lll}1 & 1 & 4.4 \\ 1 & 1 & 4.4 \\ 2 & 2 & 8.8\end{array}$

2.8

112

2.8

183 
Cuadro 3. Abundancia (N, ind) y porcentaje de la abundancia (N\%) de los anfípodos bentónicos en el sector norte del Mar Caribe.

\begin{tabular}{|c|c|c|c|}
\hline $\begin{array}{c}\text { Zona centro- norte } \\
\text { Especie }\end{array}$ & $N(N \%)$ & $\begin{array}{l}\text { Zona sur } \\
\text { Especie }\end{array}$ & $N(N \%)$ \\
\hline 1. Chevalia sp. & $8(47.1)$ & 1. Leucothoe sp. & $9(21.4)$ \\
\hline 2. Ericthonius sp. & $5(29.4)$ & 2. Grandidierella sp. & $6(14.3)$ \\
\hline 3. Ampelisca sp. & $1(5.9)$ & 3. Ampelisca holmesi & $4(9.5)$ \\
\hline 4. Ampelisca verrilli & $1(5.9)$ & 4. Leucothoe spinicarpa & $4(9.5)$ \\
\hline 5. Cerapus sp. & $1(5.9)$ & 5. Elasmopus rapax & $3(7.1)$ \\
\hline \multirow[t]{14}{*}{ 6. Concarnes sp. } & $1(5.9)$ & 6. Elasmopus sp. & $2(4.8)$ \\
\hline & & 7. Ampelisca bicarinata & $2(4.8)$ \\
\hline & & 8. Ericthonius sp. & $2(4.8)$ \\
\hline & & 9. Lysianopsis alba & $2(4.8)$ \\
\hline & & 10. Ampelisca vadorum & $1(2.4)$ \\
\hline & & 11. Bemlos sp. & $1(2.4)$ \\
\hline & & 12. Haploops sp. & $1(2.4)$ \\
\hline & & 13. Maera cf. jerrica & $1(2.4)$ \\
\hline & & 14. Gitana dominica & $1(2.4)$ \\
\hline & & 15. Rudilemboides naglei & $1(2.4)$ \\
\hline & & 16. Cerapus sp. & $1(2.4)$ \\
\hline & & 17. Dulichiella appendiculata & $1(2.4)$ \\
\hline & & 18. Lysianopsis sp. & $1(2.4)$ \\
\hline & & 19. Synopia sp. & $1(2.4)$ \\
\hline
\end{tabular}

sp. (Aoridae), Ampelisca holmesi (Ampeliscidae), Leucothoe spinicarpa (Leucothoidae) y Elasmopus rapax (Melitidae) con ca. 63\% (Cuadro 3).

El valor de $\chi_{c}^{2}$ entre las zonas sur y centro-norte en el NMC fue de $45.167\left(H_{0}: \mathrm{ZS}=\mathrm{ZC}-\mathrm{N}\right)$ rechazándose la hipótesis nula $\left(\chi_{\mathrm{c}}^{2}=45.167 \geq \chi_{\mathrm{t}}^{2}=42.976\right)$ y dando por sentado que existe un discontinuo o independencia entre la ZC-N y la ZS con base en el número de especies y su abundancia total.

\section{Discusión}

Ampliaciones geográficas y registros nuevos de anfípodos en el NMC. De la taxocenosis reconocida en este estudio, 4 especies ampliaron su distribución geográfica en el MC (Cuadro 4). Estas especies se han reconocido previamente sólo en aguas someras $(<200 \mathrm{~m})$ para las costas de Dominica (Thomas y Barnard, 1990), Colombia (Ortiz y Lemaitre, 1994) y Venezuela (Díaz-Díaz, 2001). En este estudio las especies Ampelisca bicarinata (53 m), Ampelisca holmesi (43 m), Rudilemboides naglei (45 m) y Gitana dominica (112 m) ampliaron su distribución geográfica hasta el NMC (Cuadro 4).

Para el MC y NMC se establecieron 3 registros nuevos de anfípodos pertenecientes a la familia Ampeliscidae. Ampelisca vadorum tiene una distribución a lo largo de la costa este de América del Norte, desde el SO del Golfo de San Lorenzo hasta Florida (LeCroy, 2001) y en el Golfo de México (Escobar-Briones y Winfield, 2003). La presencia de esta especie en la ZS (112 m de profundidad, NE de BC) constituye el primer registro de esta especie para el NMC. La especie Ampelisca verrilli (120 m de profundidad, sotavento de IC), recolectada en la $\mathrm{ZC}-\mathrm{N}$ representa asimismo un registro nuevo en la región. La distribución de esta especie se ha documentado para la costa oriental de los Estados Unidos y en el Golfo de México (LeCroy, 2001, Escobar-Briones y Winfield, 2003).

El género Haploops que incluye 15 especies reconocidas mundialmente y caracterizadas como especies cosmopolitas, tiene una incidencia mayor en las aguas templadas y frías de profundidades que van de 6 a >1000 $\mathrm{m}$ de profundidad (Barnard y Karaman, 1991). Mills (1971) registró a la especie Haploops setosa Boeck, 1871 en el talud continental en el sector oriental de Florida. La presencia de Haploops sp. (58 $\mathrm{m}$ de profundidad, SE de BC) en la ZS del NMC representó en este estudio un registro nuevo para la región.

Composición y dominancia en la asociación de anfípodos. 
Cuadro 4. Especies de anfípodos bentónicos con ampliación geográfica en el sector norte del Mar Caribe. Se incluye la región del Mar Caribe registrada, el intervalo de profundidad (metros) y la referencia.

\begin{tabular}{llll}
\hline \multicolumn{1}{c}{ Especies } & \multicolumn{1}{c}{$\begin{array}{c}\text { Mar } \\
\text { Caribe }\end{array}$} & $\begin{array}{c}\text { Prof. } \\
(\mathrm{m})\end{array}$ & \multicolumn{1}{c}{ Autores } \\
\hline $\begin{array}{l}\text { 1. Ampelisca } \\
\text { bicarinata }\end{array}$ & $\mathrm{V}, \mathrm{C}$ & $<200$ & $\begin{array}{l}\text { Barnard, 1954; } \\
\text { Díaz-Díaz, 2001 }\end{array}$ \\
$\begin{array}{l}\text { 2. Ampelisca } \\
\text { holmesi }\end{array}$ & $\mathrm{C}$ & $<200$ & $\begin{array}{l}\text { Ortiz y Lemaitre, } \\
1994\end{array}$ \\
$\begin{array}{l}\text { 3. Rudilemboides } \\
\text { naglei }\end{array}$ & $\mathrm{V}$ & $<200$ & $\begin{array}{l}\text { Díaz-Díaz, 2001 } \\
\begin{array}{l}\text { 4. Gitana } \\
\text { dominica }\end{array}\end{array}$ \\
\hline
\end{tabular}

$\mathrm{V}=$ Venezuela $; \mathrm{C}=$ Colombia $; \mathrm{D}=$ Dominica

Los anfípodos asociados a los arrecifes de coral constituyen uno de los grupos con mayor diversidad de especies y con adaptaciones complejas en sus ciclos biológicos. Los requerimientos de microhábitats específicos les han permitido ocupar y coexistir en formas diferentes. Por lo anterior, los patrones de composición comúnmente descritos en ambientes arrecifales y para fauna críptica incluyen estrategias funcionales de horadadoras $\mathrm{y}$ encostrantes (véase Aoridae, Amphitoidae, Corophiidae, Ischyroceridae y Melitidae), tubícolas (véase Ampeliscidae y Corophiidae), excavadoras (véase Haustoriidae, Platyischnopidae, Phoxocephalidae, Oedicerotidae y Melphidippidae) y comensales (véase Anamixidae, Iphimediidae, Leucothoidae, Sebidae y Colomastigidae) (Thomas, 1997; LeCroy, 2000).

Los anfípodos se han clasificado con base en el comportamiento reproductivo, morfología y estilo de vida (Bousfield y Shih, 1994; Bousfield, 2001) en 2 grandes grupos: Natantia-lanceolata y Reptantia-lineata. Con base en esta clasificación, de las 23 superfamilias reconocidas en el mundo 19 (82.6\%) están documentadas para el ambiente marino. En este estudio, las 7 superfamilias representan el $36.8 \%$ de la estimación mundial. El grupo Reptantia presentó el $57 \%$ en el NMC con el número mayor de superfamilias (Corophioidea, Gammaroidea, Leucothoidea y Stenothoidea) y el grupo Natantia (Ampeliscoidea, Lysianassoidea y Synopioidea) representó el $43 \%$.

La diversidad baja en niveles mayores a los de jerarquía de familia y la proporción similar de los grupos Reptantia: Natantia (4:3) para la región se explica por medio de un muestreo limitado. El de este estudio es uno de los pocos muestreos sistematizados para recolectar anfípodos crípticos, tanto para profundidades someras como por debajo de la isóbata de los 200 metros. La diversidad alta en anfípodos de sistemas arrecifales se atribuye, entre otros factores, a la complejidad y heterogeneidad del hábitat, al número elevado de microhábitats y a las adaptaciones biológicas de los anfípodos (Thomas, 1993b, 1997). El uso de estrategias selectivas en los muestreos es importante en la definición de la proporción entre componentes de ambos grupos y conlleva el dar predominio a especies de las formas avanzadas (Reptantia) y con estrategias de vida complejas (por ejemplo, Anamixidae [sensu Barnard y Karaman 1991], Colomastigidae, Leucothoidae [sensu Barnard y Karaman 1991] ) los resultados de este estudio contribuyen con esta información para un sector del sistema arrecifal del NMC.

De las 925 especies de anfípodos registradas para México hasta el año 2004 (11.5\% de la cifra mundial estimada), el NMC representaba el 19\% con 179 especies (Cuadro 5). Los caprélidos y gammáridos constituían en dicho estudio el $13.3 \%$ nacional, con 123 especies reconocidas (McKinney, 1977, 1979; Oliva-Rivera, 1998, 2003; Escobar-Briones et al., 2002). Este estudio reconoció un incremento de especies (23) con respecto al número total de especies de anfípodos registradas, aumentando en $3.5 \%$ el porcentaje mundial relativo para el NMC.

Patrones de distribución espacial. Los arrecifes de coral representan uno de los ecosistemas con mayor diversidad taxonómica (Reaka-Kudla, 2001) y complejidad espacial (Jordán-Dahlgren, 1993) debido a la variedad de macro y microhábitats (por ejemplo: corales, algas, praderas marinas, invertebrados sésiles, fragmentos de coral [Thomas, 1993b]). La variación de las condiciones de energía en estos ambientes sedimentarios se ha relacionado con el oleaje, la circulación superficial-subsuperficial, las perturbaciones atmosféricas y la geomorfología local (Gutiérrez-Carbonell et al., 1993), que ocasiona la redistribución de los sedimentos, de los cabezos de coral (Nolasco-Montero y Carranza-Edwards, 1988) y de los organismos (Thomas, 1993a). Como resultado de la capacidad amortiguadora (Jordán-Dahlgren, 1993) y promotora del desarrollo de diversidad (Thomas, 1993a) de los arrecifes, la variabilidad en el número y patrones de distribución de las especies que concurren en éstos y en los ambientes vecinos sedimentarios depende de la intensidad de la matriz ambiental local (Jordán-Dahlgren, 1993).

Los arrecifes se encuentra sujetos al efecto de los huracanes y ciclones que fracturan y depositan los fragmentos de coral y agregados de algas a los canales profundos transportando a la fauna asociada a profundidades mayores. El efecto es local y la permanencia de esta fauna depende de la cantidad de alimento disponible; así, la agregación de los organismos se explica por estos 
Cuadro 5. Número de especies de anfípodos registrados y porcentaje relativo para México: Océano Pacífico (OP), Golfo de México (GM), sector norte del mar Caribe (NMC) y el continente $(\mathrm{C})$.

\begin{tabular}{cccccc}
\hline Suborden & OP & GM & NMC & C & Total \\
\hline Gammaridea & 280 & 200 & 110 & 39 & 629 \\
Caprellidea & 9 & 15 & 13 & 0 & 37 \\
Ingolfiellidea & 0 & 1 & 0 & 0 & 1 \\
Hyperiidea & 143 & 59 & 56 & 0 & 258 \\
Total & 432 & 275 & 179 & 39 & 925 \\
Porcentaje & $46 \%$ & $30 \%$ & $19 \%$ & $4 \%$ & $100 \%$ \\
\hline
\end{tabular}

Tomado y modificado de Escobar-Briones et al. (2002) y

Escobar-Briones y Winfield (2003).

eventos.

La velocidad de corriente en el canal que separa las islas del macizo continental es de $\sim 0.5 \mathrm{a} 1 \mathrm{~m} \cdot \mathrm{s}^{-1}$ y genera ondulitas en el sedimento de la porción central del canal con falta de consolidación del sustrato, predominantemente arenoso (Graus y MacIntyre, 1989). La talla de la fauna $(<1 \mathrm{~cm})$ y los hábitos crípticos y de enterramiento de ésta en sustratos de baja movilidad sugieren que el patrón de corrientes y la velocidad tiene un efecto mínimo sobre el patrón de distribución. El depósito de cabezos se reconoce predominantemente en la base del escarpe tanto del Borderland como del sector oriental de la península, zona que tiene una afectación menor por el patrón de corrientes del canal. Por lo anterior, es de esperar que los patrones de distribución, de riqueza de especies y de agregación reconocidos en aguas someras se verán reflejados en los cabezos de coral y en aguas profundas por la agregación de algas y fragmentos en el fondo. Este patrón de ocurrencia se fundamenta en que el cabezo y los fragmentos de coral son refugios para los anfípodos evitando depredadores y corrientes que los transporten. La diversidad varía localmente con el tiempo y con el aporte de material alimenticio y competencia por espacio en los fragmentos.

El número de especies y composición varían a lo largo de un gradiente de sur a norte que se ha atribuido al desarrollo del sistema arrecifal y procesos asociados a la hidrodinámica y clima. La parte frontal del arrecife de la ZC-N presenta un desarrollo reducido que se relaciona a la acumulación de sedimentos por efecto de ciclones y tormentas (Jordán-Dahlgren, 1979, 1989b, 1993). El número reducido de especies de anfípodos en esta zona ZC-N y la distribución limitada en sotavento y NE de la IC se explican por la presencia de un desarrollo coralino que protege del embate de las tormentas. La riqueza mayor de especies (19 spp.) se registró en la ZS y se relacionó con el incremento en la complejidad-heterogeneidad estructural del arrecife y un mayor depósito de fragmentos y cabezos coralinos, particularmente en el SE y SO. Esta zona arrecifal se ha definido por su desarrollo amplio e incremento en la heterogeneidad estructural en la cresta y hasta la parte frontal, con formación de macizos y canales en proceso intenso de acreción y una frecuencia relativa menor de perturbaciones atmosféricas (Jordán-Dahlgren, 1989a, 1993). Asimismo, en el SE-E del banco Chinchorro se ha reconocido la presencia de praderas marinas y camas de algas (Jordán-Dahlgren y Martin, 1988; Oliva-Rivera, 2003) que proveen microhábitats y sustratos para anfípodos. Los resultados de este estudio sugieren la necesidad de continuar los muestreos sistematizados y selectivos a cada hábitat del sistema arrecifal a fin de validar el patrón de distribución propuesto en este trabajo.

\section{Agradecimientos}

A la Dirección General de Asuntos del Personal Académico (DGAPA-UNAM) IN224503 y al Consejo Nacional de Ciencia y Tecnología (CONACYT) 40158 por el apoyo otorgado. A la tripulación del Edwin Link, J.L. Villalobos, IBUNAM y L.A. Soto, ICML por su ayuda en la fragmentación del material y extracción de organismos a bordo. Los resultados corresponden a la colaboración del Instituto de Ciencias del Mar y Limnología-UNAM (EEB) con Harbor Branch Oceanographic Institution (HBOI).

\section{Literatura citada}

Barnard, J. L. 1954. Amphipoda of the family Ampeliscidae collected by the Velero III in the Caribbean Sea. Allan Hancock Atlantic Expedition 7:1-12.

Barnard, J. L. 1964. Deep-sea Amphipoda (Crustacea) collected by the R/V "Vema" in the eastern Pacific Ocean and the Caribbean and Mediterranean Seas. Bulletin of the American Museum of Natural History 127:1-46.

Barnard, J. L. y G. S. Karaman. 1991. The families and genera of marine Gammaridean Amphipoda (except marine Gammaroids). Records of the Australian Museum, Supplement 13, parts I and II, 866 p.

Barnard, J. L. y J. D. Thomas. 1983. A new species of Amphilochus from the gorgonian Pterogorgia anceps in the Caribbean Sea. In Selected Papers on Crustacea, P. A. John (ed.). Trivandrum: The Aquarium, Washington, p.179-187.

Bellan-Santini, D. 1999. Ordre des Amphipodes (Amphipoda Latreille, 1816). In Traite de Zoologie. Anatomie, Systématique, Biologie de Crustacés Peracarides, tome VII. Fascicule IIIA, P. Grassé (ed.). 
Paris, p. 95-168.

Bousfield, E. L. 1973. Shallow-water gammaridean Amphipoda of New England. Cornell University Press, Ithaca, New York. 312 p.

Bousfield, E. L. 2001. An updated commentary on phyletic classification of the amphipod Crustacea and its applicability to the North American fauna. Amphipacifica 3:49-119.

Bousfield, E. L. y C. T. Shih. 1994. The phyletic classification of amphipod crustaceans: problems in resolution. Amphipacifica 1:76-134.

Brower, J., J. Zar y C. Ende. 1998. Field and laboratory methods for general ecology. McGraw-Hill, New York. 489 p.

Bunge, L., J. Ochoa, A. Badan, J. Candela y J. Sheinbaum. 2002. Deep flows in the Yucatan Channel and their relation to changes in the Loop Current extension. Journal of Geophysical Research 107(C12):3233, doi:10.1029/2001JC001256.

Burton, G. A., Jr. 2002. Sediment quality criteria in use around the world. Limnology 3:65-75.

Candela, J., J. Sheinbaum, J. Ochoa, A. Badan y R. Leben. 2002. The potential vorticity flux through the Yucatan Channel and the Loop Current in the Gulf of Mexico. Geophysical Research Letters 29:2059, doi:10.1029/ 2002 GL015587.

Cartes, J. E., M. Elizalde y J. C. Sorbe. 2001. Contrasting life-histories, secondary production, and trophic structure of peracarid assemblages of the bathyal suprabenthos from the Bay of Biscay (NE Atlantic) and the Catalan Sea (NW Mediterranean). Deep-Sea Research I 48:2209-2232.

Cházaro-Olvera, S., I. Winfield, M. Ortiz y F. Álvarez. 2002. Peracarid crustaceans from three inlets in the southwestern Gulf of Mexico: new records and range extensions. Zootaxa 123:1-16.

Díaz-Díaz, Y. J. 2001. Contribución al conocimiento del orden Amphipoda de la costa centro-occidental de Venezuela. Tesis de Maestría, Universidad Simón Bolívar, Caracas. 543 pp.

Escobar-Briones, E. 2004. Structure and function in the ecosystems of the intra-Americas sea (IAS). In The sea, A. Robinson y K. Brink (eds.). Harvard College, p. 225-258.

Escobar-Briones, E. e I. Winfield. 2003. Checklist of the benthic Gammaridea and Caprellidea (Crustacea: Peracarida: Amphipoda) from the Gulf of Mexico continental shelf and slope. Belgian Journal to Zoology 133:37-44.

Escobar-Briones, E., I. Winfield, M. Ortiz, R. Gasca y E. Suárez. 2002. Amphipoda. In Biodiversidad, taxonomía y biogeografía de artrópodos de México.
Hacia una síntesis de su conocimiento, vol. III. 1a. Ed. J. Llorente y J. J. Morrone (eds.). Comisión Nacional para el Conocimiento y Uso de la Biodiversidad y Universidad Nacional Autónoma de México, p. 342371.

Gallegos, A. y S. Czistrom. 1997. Aspectos de la oceanografía física regional del Mar Caribe. In Contribuciones a la oceanografía física en México, M. F. Lavin (ed.). Monografía 3, Unión Geofísica Mexicana, México. D.F. p. 225-242.

Galan, A. 1984. A systematic study of Amphipoda (Crustacea) of the Caribbean coast of Venezuela. Ph.

D. Thesis, Department of Pure and Applied Biology, Imperial College of Science and Technology, London University, London. 422 p.

Graus, R. R. y I. G. MacIntyre. 1989. The zonation patterns of Caribbean coral reefs as controlled by wave and energy input, bathymetric setting and reef morphology: computer simulation experiments. Coral Reefs 8:9-18.

Guilcher, A. 1988. Coral reef geomorphology. Wiley, New York. 228 p.

Gutiérrez-Carbonell, D., C. García-Sáenz, M. Lara-Pérez, C. Padilla-Souza, J. Pizaña-Alonso y R. MacíasOrdóñez. 1993. Caracterización de los arrecifes coralinos de la Reserva de la Biosfera Sian Ka'an, Q. Roo. Serie Documentos Sian Ka'an, Quintana Roo. Gobierno del Estadode Quintara Roo. 47 p.

Jordán-Dahlgren, E. 1979. Estructura y composición de arrecifes coralinos en la región noreste de la Península de Yucatán, México. Anales del Instituto de Ciencias del Mar y Limnología, Universidad Nacional Autónoma de México 6:69-86.

Jordán-Dahlgren, E. 1989a. Efecto de la morfología del sustrato en el desarrollo de la comunidad coralina. Anales del Instituto de Ciencias del Mar y Limnología, Universidad Nacional Autónoma de México 16:105118.

Jordán-Dahlgren, E. 1989b. Gorgonian community structure and reef zonation patterns on Yucatán coral reefs. Bulletin of Marine Science 45:678-696.

Jordán-Dahlgren, E. 1993. El ecosistema arrecifal coralino del Atlántico mexicano. Revista de la Sociedad Mexicana de Historia Natural, Vol. Esp: 157-175.

Jordán-Dahlgren, E. y M. Martin. 1988. Chinchorro: morphology and composition of a Caribbean Atoll. Atoll Research Bulletin 310:112-120.

Krapp-Schickel, T. y N. E. Jarrett. 2000. The amphipod family Melitidae on the Pacific coast of North America: Part II. The maera-ceradocus complex. Amphipacifica 2:23-61.

LeCroy, S. 2000. An illustrated identification guide to the nearshore marine and estuarine gammaridean 
Amphipoda of Florida. Families Gammaridae, Hadziidae, Isaeidae, Melitidae and Oedicerotidae, vol. 1. U.S. Environmental Protection Agency. WM724. $195 \mathrm{p}$.

LeCroy, S. 2001. An illustrated identification guide to the nearshore marine and estuarine gammaridean Amphipoda of Florida. Families Ampeliscidae, Amphilochidae, Ampithoidae, Aoridae, Argissidae and Haustoriidae, vol. 2. U.S. Environmental Protection Agency. WM724. 197-410 p.

Lowry, J.K. y H.E. Stoddart. 1997. Amphipoda Crustacea IV.Families Aristiidae, Cyphocarididae, Endevouridae, Lysianassidae, Scopelocheiridae, Uristidae. Memoirs of the Hourglass Cruises 10:1-148.

Markham, J. C., F. E. Donath, J. L. Villalobos y A. C. Díaz. 1990. Notes on the shallow-water marine Crustacea of the Caribbean coast of Quintana Roo, Mexico. Anales del Instituto de Biología, Universidad Nacional Autónoma de México, Serie Zoología 61:405-446.

McKinney, L. D. 1977. The origin and distribution of shallo water gammaridean Amphipoda in the Gulf of Mexico and Caribbean Sea with notes on their ecology. Ph. D. thesis, Texas A\&M University, Austin. 400 p.

McKinney, L. D. 1979. Liljeborgiid amphipods from the Gulf of Mexico and Caribbean Sea. Bulletin of Marine Science 29:140-154.

Merino-Ibarra, M. 1986. Aspectos de la circulación costera superficial del Caribe mexicano con base en observaciones utilizando tarjetas de deriva. Anales del Instituto de Ciencias del Mary Limnología, Universidad Nacional Autónoma de México 13:31-46.

Mills, E. L. 1971. Deep-sea Amphipoda from the western North Atlantic Ocean. The family Ampeliscidae. Limnology and Oceanography 16:357-355.

Nolasco-Montero, E. y A. Carranza-Edwards. 1988. Estudio sedimentológico regional de playas de Yucatán y Quintana Roo, México. Anales del Instituto de Ciencias del Mar y Limnología, Universidad Nacional Autónoma de México 15:49-66.

Oliva-Rivera, J. J. 1998. Anfípodos. In Enciclopedia de Quintana Roo, tomo I A-B, X. Maiza (ed.). Gobierno del Estado de Quintana Roo, Chetumal. p. 148-169.

Oliva-Rivera, J. J. 2003. The Amphipod fauna of Banco Chinchorro, Quintana Roo, Mexico with ecological notes. Bulletin of Marine Science 73:77-89.

Oliva-Rivera, J. J. y M. S. Jiménez-Cueto. 1992. Anfípodos bentónicos (Crustacea: Peracarida) de la Reserva de la Biosfera de Sian Ka'an, Quintana Roo, México. In Diversidad biológica de la Reserva de la Biosfera de Sian Ka'an, Quintana Roo, México, vol. II D. Navarro y E. Suárez-Morales (eds.). CIQRO/SEDESOL, Chetumal, Quintana Roo. p. 170-195.
Oliva-Rivera, J. J. y M. S. Jiménez-Cueto. 1997. Composición, distribución y abundancia de los crustáceos 7peracáridos de la Laguna de Yalahau, Quintana Roo. Avacient 23:26-31.

Ortiz, M. 1994. Clave gráfica para la identificación de familias y géneros de anfípodos del suborden Gammaridea del Atlántico Occidental tropical. Anales del Instituto de Investigaciones Marinas Punta Betín 23:59-101.

Ortiz, M. y R. Lemaitre. 1994. Crustáceos anfípodos (Gammaridea) colectados en las costas del Caribe colombiano, al sur de Cartagena. Anales del Instituto de Investigaciones Marinas Punta Betín 23:119-127.

Reaka-Kudla, M. L. 2001. Arrecifes de coral: biodiversidad y conservación. In Enfoques contemporáneos para el estudio de la biodiversidad, H. M., Hernández, A. N. García-Aldrete, F. Álvarez y M. Ulloa (eds.). Instituto de Biología, Universidad Nacional Autónoma de México. México, D. F. p. 221-243.

Ruffo, S. 1950. Studi sui crostacei anfipodi. XXII. Anfipodi del Venezuela raccolti dal dott. G. Marcuzzi. Memorie del Museo Cívico di Storia Naturale di Verona 2:4965.

Ruffo, S. 1954. Studi sui crostacei anfipodi XL. Nuovi anfipodi raccolti nel Venezuela. Memorie del Museo Cívico di Storia Naturale di Verona 4:117-125.

Shoemaker, C. 1933. Amphipoda from Florida and the West Indies. American Museum Novitates 598:1-24.

Shoemaker, C. 1934. Reports on the collections obtained by the first Johnson-Smithsonian deep-sea expedition to the Puerto Rican deep. Three new amphipods. Smithsonian Miscellaneous Collections 91:1-6.

Shoemaker, C. 1935. The amphipods of Porto Rico and the Virgin Islands. Science Survey of Porto Rico and the Virgin Islands. New York Academy of Science 15:229-253.

Shoemaker, C. 1948. The Amphipoda of the SmithsonianRoebling Expedition to Cuba in 1937. Smithsonian Miscellaneous Collections 110:1-15.

Sokal, R. y F. J. Rohlf. 1988. Biometría. interamericana, México. 587 p.

Stephensen, K. 1933. Amphipoda from the marine salines of Bonaire and Curacao. Zoologische Ergebnisse ainer Reise nach Bonaire, Curacao und Aruba im Jahre 1930. Zoologische Jahrbucher Systematik 64:437-446.

Stephensen, K. 1947. Amphipods from Curacao, Bonaire, Aruba and Margarita. Studies on the Fauna of Curacao, Aruba, Bonaire and the Venezuelan Islands 11:1-20.

Thomas, J. D. 1993a. Biological monitoring and tropical biodiversity in marine environments: a critique with recommendations, and comments on the use of amphipods as bioindicators. Journal of Natural History 
27:795-806.

Thomas, J. D. 1993b. Identification manual for marine Amphipoda (Gammaridea): I. Common coral reef and rocks, bottom amphipods of South Florida. Final Report DEP, contract number SP290. Smithsonian Institution, Washington, D.C.

Thomas, J. D. 1997. Systematics, ecology and phylogeny of the Anamixidae (Crustacea: Amphipoda). Records of the Australian Museum 49:35-98.
Thomas, J. D. y J. L. Barnard. 1990. Gitana dominica, a new species from the Caribbean Sea (Amphipoda: Amphilochidae). Proceedings of the Biological Society of Washington 103:617-623.

Thomas, J. D. y M. Ortiz. 1995. Leucothoe laurensi, a new species of leucothoid amphipod from Cuban waters (Crustacea, Amphipoda, Leucothoidae). Proceedings of the Biological Society of Washington 108:613616. 